\title{
TOWARDS AN AUTOMATED GEOREFERENCING AND ORTHORECTIFICATION OF ARCHAEOLOGICAL AERIAL PHOTOGRAPHS
}

\author{
M. Doneus, G. Verhoeven, W. Karel, C. Briese, N. Pfeifer, C. Ressl
}

\section{INTRODUCTION}

Aerial photography is one of the oldest archaeological prospection methods. Although it is highly efficient in detecting sites, its full potential (i.e. the detailed mapping of archaeological and palaeoenvironmental structures) can only be used when the photographs are georeferenced, (ortho)rectified, interpreted and mapped. Georeferencing, also known as image orientation or registration, assigns spatial information to the imagery to explicitly define their location and rotation in respect to a specific Earth-fixed coordinate frame. This enables their transcription to a map, a process that obviously involves the archaeological interpretation of the detected structures and/or features in relation to other available georeferenced data sets (Palmer, 2000). When executed repetitively, several multi-temporal observations can be mosaicked into an extensive overall view of an archaeological region, which will be used as basic information for further prospection, excavation, protection measures, and spatial analysis.

Irrespective of the method applied, the georeferencing of the images is commonly determined with ground control points (GCPs), whose measurement and identification is a timeconsuming operation (that is only partly reduced in photogrammetric packages by using automatic tie points and a block adjustment for multiple images). Especially in large-scale archaeological projects with thousands of images, this is currently considered to be the biggest bottleneck. Along with specialised software, reference data, photogrammetric skills and experience are required. Therefore, aerial archaeology often does not go beyond the data acquisition stage. As a result, millions of aerial photographs (APs) are currently stored in archives that often only allow a very limited geometric access of the imagery. These archives contain an enormous amount of valuable optical information for detecting and documenting archaeological landscapes, which cannot be exploited efficiently.

\section{THE PROJECT}

Hence, automated techniques for the georeferencing of archaeological APs are highly desirable. In order to allow an absolute georeferencing, this approach can be based on the available georeferenced reference data (orthophoto and digital terrain model or DTM). Within this workflow, however, several issues of typical archaeological APs have to be considered:

- both vertical and oblique images are acquired

- different non-metric (uncalibrated) cameras are used

- oblique photographs often lack a high number and/or good distribution of possible ground control points

- oblique APs are often shot from an extremely oblique viewpoint

- often, even rough georeferencing is missing [e.g. position information by a hand-held GNSS (global navigation satellite system) receiver].
Since March 2012, the Austrian Science Fund (FWF) is supporting the project "Automated Georeferencing and Orthorectification of Archaeological Aerial Photographs (ARAP)". Three international partner organisations (Vienna Institute of Archaeological Science of the University of Vienna, LBI for Archaeological Prospection and Virtual Archaeology based on an international partnership, and Research Groups of Photogrammetry and Remote Sensing of TU-Vienna) are co-operating within the project. They have long-term experience in archaeological prospection, especially aerial archaeology and photogrammetry. The general aim of the proposed project is to create solutions for the automated georeferencing and orthophoto generation of archaeological APs. The resulting workflow should allow the efficient processing of both newly generated and previously acquired aerial images based on available reference data.

In order to fulfil these requirements, the project will (1) evaluate a calibrated digital still camera (DSC) with an attached positioning and orientation system (POS) for archaeological prospection and automated orthophoto production = "hardware approach"; (2) develop a workflow including software for automatic orientation of APs without accurate initial positioning and rotation information = "software approach".

Both approaches will be implemented in the conventional aerial reconnaissance work from a small aeroplane as well as in an innovative method using an unmanned aerial vehicle (UAV) to acquire aerial imagery completely autonomously and being independent of conventional aircrafts.

\section{HARDWARE APPROACH}

This approach aims at creating a hand-held calibrated DSC with an attached positioning and orientation system (POS). By combining a low-cost IMU (inertial measurement unit) and a GNSS receiver, the POS observes the external orientation parameters as input for the previously outlined software approach. Currently, several affordable IMU solutions (prices around $€ 200$ ) are being tested for their accuracy, tendency to drift and consistent performance. In addition, they should allow easy integration with lowcost GNSS receivers, enable sub-second camera synchronisation and data logging (on the same board or using a third hardware component) while featuring low power consumption. Finally, the integrated POS solution must be robust enough to be taken in the air and small enough to fit in a case that is easily mountable on a camera's hot/flash shoe (Figure 1). Within the outlined software workflow (during the bundle block adjustment), the POS data will be used as initial values resp. as weighted observations of the exterior orientation of the photograph. This will allow reducing the effort for estimating these initial values. However, in order to increase the absolute accuracy of the final georeferencing, the usage of available high quality DTM and orthophotos will be essential. 


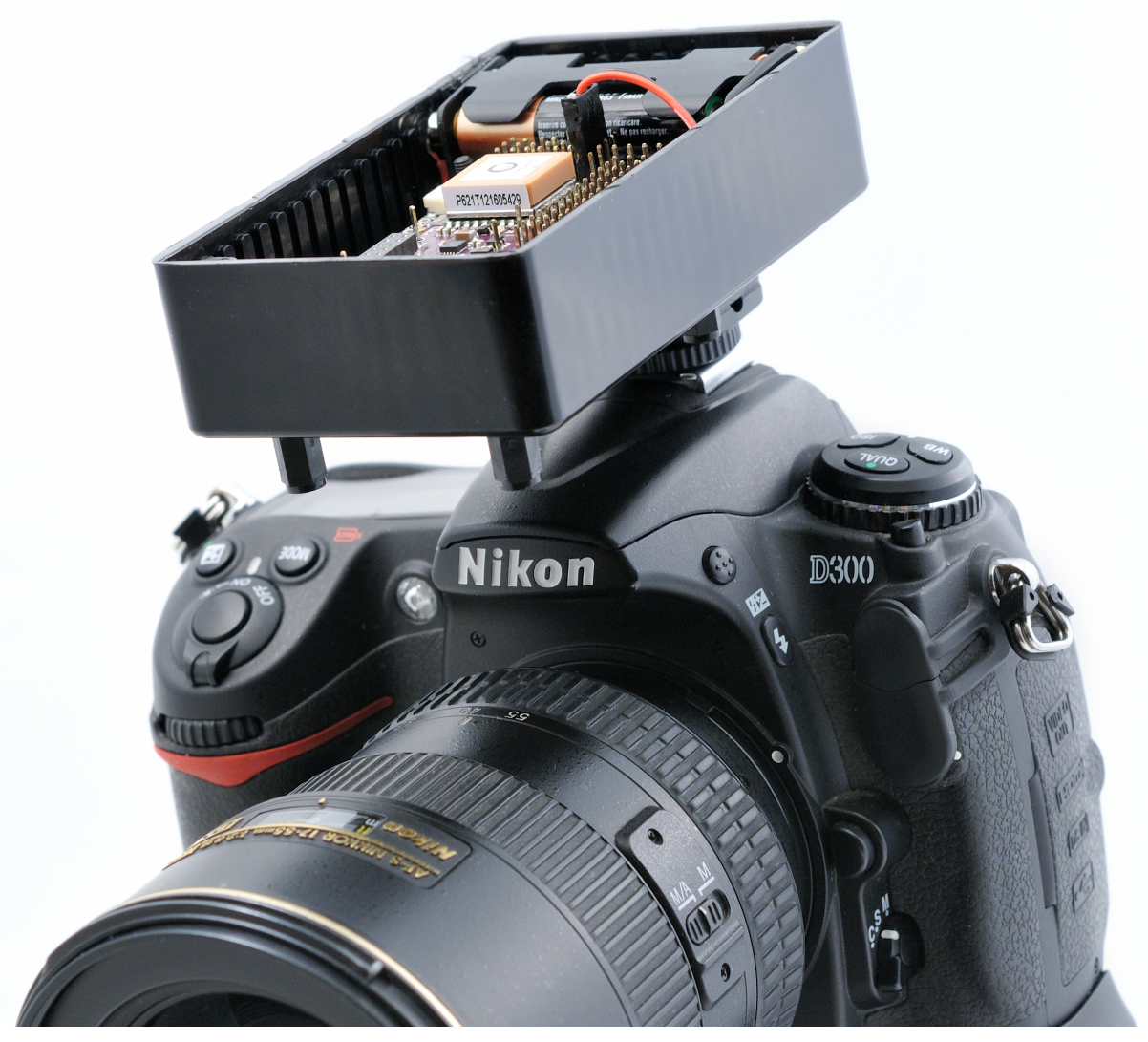

Figure 1: The first prototype of a hot/flash shoe-mounted case which holds the integrated POS solution.

\section{SOFTWARE APPROACH}

In the initial phase of the project, the software approach is vital, as it is the only method that can be used for the millions of vertical and oblique APs that are in archives waiting to be processed. Furthermore, researchers who do not have the hardware necessary for the second integrated solution could employ this software. This approach is primarily based on image matching and orthorectification algorithms, a given set of orthophotos and a digital terrain model to georeference the oblique images to existing orthophotos of the respective area.

Developments in recent years have made it possible to compute the relative orientation of photographs of a static scene in a fully automatic manner, even without initial values. Algorithms based on stable keypoints found in the combined image and scale spaces (Lowe, 2004), and the matching of corresponding image points by finding nearest neighbours in the descriptor (feature) space defined by statistics of their local neighbourhoods, have proven to cope with moderate geometric and radiometric discrepancies between corresponding image regions. Together with the relative image orientation, a sparse reconstruction of object space is generated that, however, lacks scale, position, and attitude with respect to an Earth-fixed coordinate frame.

These unknown parameters shall be determined by help of existing orthophoto maps. However, APs (to be georeferenced) and georeferenced orthophotos are generally taken at largely different calendar dates with different vegetation and infrastructure (and sometimes even different terrain elevation). As a result, the discrepancies between homologous image regions are gen- erally too large for these algorithms to succeed. To cope with this challenge, and to keep computation times reasonable, a priori knowledge must be used that narrows the search both in image and parameter spaces. For example, the external orientation parameters from the hardware approach can be used as direct input, while vegetated areas yielding unreliable keypoints may be masked out, or the principal components of the sparse point cloud may be used to estimate the rotations about the horizontal axes for flat terrain. As the number of possible combinations of information known beforehand is large, the software approach must be adaptable to various levels of processing. Thus, it will use a scripting language on the higher levels, and a flexible database scheme that supports 3-dimensional spatial geometries and queries.

\section{CASE STUDY}

The aerial archaeology unit at the Department for Prehistoric and Medieval Archaeology of the University of Vienna (UFG) houses more than 110,000 vertical and oblique APs in its aerial archive (Doneus et al., 2001). The metadata of all photographs, including their footprints, are stored in a GIS-based database (Figure 2). Since 2001, the total archive is being systematically digitized using a professional photogrammetric scanner (Vexcel Ultra Scan 5000). At the moment, roughly $60 \%$ of all aerial photographs have been digitised with a resolution of $10 \mu \mathrm{m}$ (for B\&W images) and $1510 \mu \mathrm{m}$ (for colour infrared images). From this dataset, an archaeological case study will serve to test and evaluate all proposed solutions for automated image orien- 


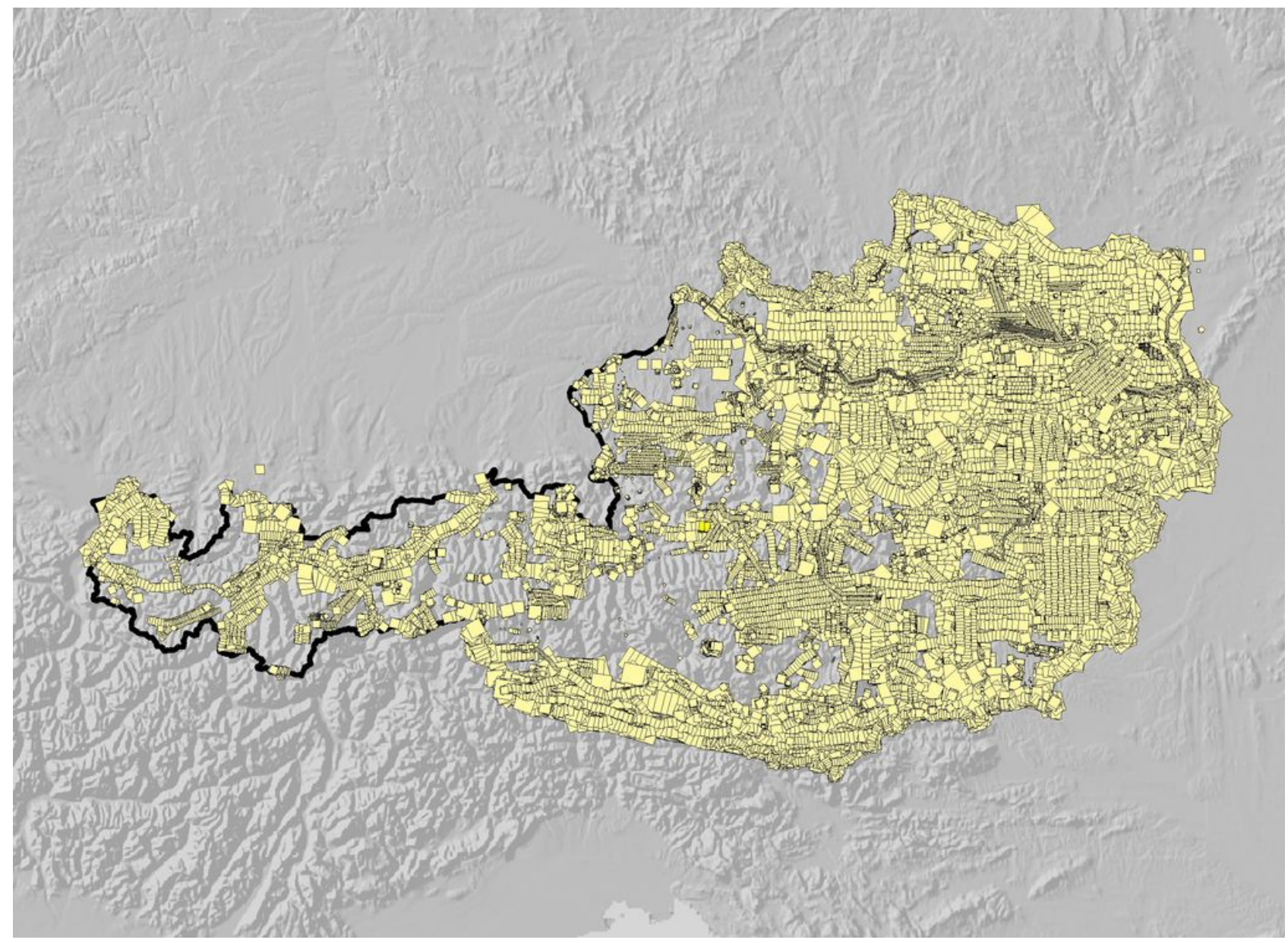

Figure 2: Map of the approximately determined footprints of more than 110,000 aerial photographs stored at the aerial archive of the Department for Prehistoric and Medieval Archaeology in Vienna.

tation and orthophoto generation. The proposed $250 \mathrm{~km}^{2}$ area is largely covered by vertical and oblique APs from various years and seasons, with scales ranging from 1:25,000 to 1:5,000. The evaluation should identify problems and improve our approach, both using conventional aeroplanes as well as UAVs. The case study should also result in written guidelines about the methods of proper aerial data acquisition and georeferencing.

\section{ACKNOWLEDGEMENTS}

This research is being carried out with the financial support of the Austrian Science Fund (FWF): P24116-N23.

\section{REFERENCES}

Doneus M, Eder-Hinterleitner A, Neubauer W. 2001. Archaeological Prospection in Austria. In Archaeological Prospection, 4th International Conference on Archaeological Prospection, Doneus M, Eder-Hinterleitner A, Neubauer W (eds.). Austrian Academy of Sciences Press: Vienna; 11-34.

LowE DG. 2004. Distinctive Image Features from ScaleInvariant Keypoints. International Journal of Computer Vision 60(2): 91-110.

Palmer R. 2000. A View From Above: Can Computers Help Aerial Survey? In On the Theory and Practice of Archaeological Computing, Lock GR, Brown K (eds.). Oxford University Committee for Archaeology: Oxford; 107-131. 